\title{
Motion-preserving, 2-stage transoral and posterior treatment of an unstable Jefferson fracture in a professional football player
}

\author{
Gerald E. Rodts Jr., MD, ${ }^{1}$ Griffin R. Baum, MD, ${ }^{1}$ Fermin G. Stewart, MD, ${ }^{2}$ and John G. Heller, MD' \\ ${ }^{1}$ The Emory Orthopaedics \& Spine Center, Emory University School of Medicine; and 2Johns Creek ENT, Atlanta, Georgia
}

\begin{abstract}
The authors report the case of a patient who suffered a Jefferson fracture during a professional football game. The C-1 (atlas) fracture was widely displaced anteriorly, but the transverse ligament was intact. In an effort to enable a return to play and avoid intersegmental (C1-2) fusion, the patient underwent a transoral approach for open reduction and internal fixation of the fracture. The associated posterior ring fracture displacement widened after this procedure, and a subsequent posterior arthrodesis and fixation of the fracture site was performed 6 months later when the fracture failed to heal with rigid collar immobilization. The approach maintained the normal range of motion at the atlantoaxial and atlantooccipital joints, which would have been sacrificed by an atlantoaxial or occipitocervical fusion, as is traditionally performed. Ultimately, the patient decided not to return to the football field, but this approach could avoid the more significant loss of motion associated with atlantoaxial or occipitocervical fusion for unstable Jefferson fractures.
\end{abstract}

https://thejns.org/doi/abs/10.3171/2017.6.SPINE17274

KEY WORDS cervical trauma; sports medicine; Jefferson fracture; C-1 (atlas) fracture; transoral approach; motion preserving; open reduction and internal fixation; ORIF

$\mathbf{J}$ EFFERSON fractures are uncommon cervical spine injuries and comprise about $2 \%$ of all cervical spine injuries. Among professional football players, not a single unstable Jefferson fracture has been recorded in the current professional football database (unpublished data). Minimally displaced Jefferson fractures with an intact transverse ligament can typically be treated in a cervical collar, with a high rate of osseous union across the fracture lines. Fractures that are more displaced can be associated with disruption of the transverse ligament and even other ligaments associated with the atlantoaxial and atlantooccipital joints and clivus. $\mathrm{C}-1$ fractures that are displaced more than $7 \mathrm{~mm}$ and are accompanied by rupture of the transverse ligament have been termed unstable, with a higher rate of nonunion and chronic occiput$\mathrm{C} 1$ and/or C1-2 instability. In these cases, Halo brace immobilization or potentially surgical stabilization is often used. Classically, unstable Jefferson fractures are treated with various types of posterior atlantoaxial fusions or occipitocervical fusion techniques. There are only 2 publications in the peer-reviewed literature pertaining to anterior fixation of unstable Jefferson fractures. Another report described a posterior-only approach for a motion-sparing fixation of a Jefferson fracture. ${ }^{1-3}$ Although some surgeons have extensive experience with transoral resection of the odontoid, that surgery typically involves only bone and/or pannus resection and not any type of osteosynthesis or arthrodesis. The transoral approach for rheumatoid disease, for example, requires a much narrower corridor than that required for open reduction and internal fixation (ORIF) of a displaced C-1 arch fracture. The transoral approach for fixation of traumatic fracture-dislocations of the C1-2 complex is technically challenging, and there are no specific instrumentation sets or spinal implants designed for treating these types of fractures via a transoral approach. We report the case of a patient who suffered a Jefferson fracture during a professional football game and the subsequent combined transoral ORIF and posterior minimally invasive ORIF of the fracture. The goal was to preserve craniocervical motion, specifically to avoid a fusion, which would have precluded the patient's return to play in professional football.

\section{Case Report}

History and Physical Examination

The Emory institutional review board approved the review of the patient's records, and patient consent was ob-

ABBREVIATIONS AADI = atlanto-axial-dens interval; $A P=$ anteroposterior; $H R-Q O L=$ health-related quality of life; $O R I F=$ open reduction and internal fixation . SUBMITTED March 5, 2017. ACCEPTED June 28, 2017.

INCLUDE WHEN CITING Published online December 1, 2017; DOI: 10.3171/2017.6.SPINE17274. 
tained. The patient was a 23-year-old man who developed axial neck pain and occipital pain following helmet-tobody contact during a professional football game. There was no loss of consciousness. The patient did not report any radicular pain, paresthesias, motor weakness, imbalance, or loss of fine motor coordination. Neurological examination revealed normal mental status, normal cranial nerve findings, and normal motor, sensory, fine motor, reflex, and gait function.

A CT scan demonstrated a midline C-1 anterior arch fracture with $12 \mathrm{~mm}$ of separation and a right paramedian posterior arch fracture that was 1-2 mm displaced (Fig. $1 \mathrm{~A}-\mathrm{C})$. The patient demonstrated complete loss of lordosis on supine sagittal CT images (Fig. 1D). No subaxial traumatic injuries were noted. No comminution or avulsion of bone was seen at the insertion of the transverse ligament along the medial aspect of the $\mathrm{C}-1$ lateral masses. Axial T1- and T2-weighted MR images showed an intact transverse ligament (Fig. 2). No spinal cord contusion or edema was identified.

\section{Surgical Decision Making}

Several options were presented to the patient and his team of physicians. The first and most conservative option was Halo vest or rigid cervical collar immobilization and observation. If the fracture sites could heal with external immobilization alone, the $\mathrm{Cl}-2$ complex might remain competent since the transverse ligament was preserved. The marked diastasis of the anterior component of the C-1 arch fracture, however, was judged to profoundly increase the rate of nonunion with Halo vest or cervical collar immobilization alone. If bracing were attempted and failed as expected, then posterior fusion for this unstable fracture would be recommended.

The second option was posterior atlantoaxial fusion, or potentially an occipitocervical fusion. For most patients, C1-2 posterior fusion is a valid option, and although it does result in a $40 \%$ lateral rotation, there are few changes in health-related quality of life (HR-QOL) outcomes for most patients. An occipitocervical fusion does more severely reduce cervical range of motion and has more deleterious effects on HR-QOL. After either of these interventions, however, return to play in professional football would not be possible due to loss of range of motion following fusion at $\mathrm{C} 1-2$ or occiput-C1-2. Furthermore, there would be incalculable potential risk to the adjacent levels when subjected to axial loading extension or flexion or extension injuries with full contact.

A third option that could increase the rate of primary healing of the anterior and posterior fractures and that could theoretically result in minimal loss of lateral rotation was ORIF of the C-1 fracture. With the transverse ligament intact, a surgical procedure could achieve a healed anatomical result equivalent to that of a lesser, nondisplaced-type fracture that heals with brace immobilization. Since the wide displacement involved the anterior arch in the midline, a standard transoral approach could provide access to the fracture site for ORIF. The posterior motionpreserving approach described by $\mathrm{Hu}$ et al. was thought to not be effective enough to reduce the large anterior diastasis of the midline arch of $\mathrm{C}-1 .{ }^{1} \mathrm{~A}$ direct and complete

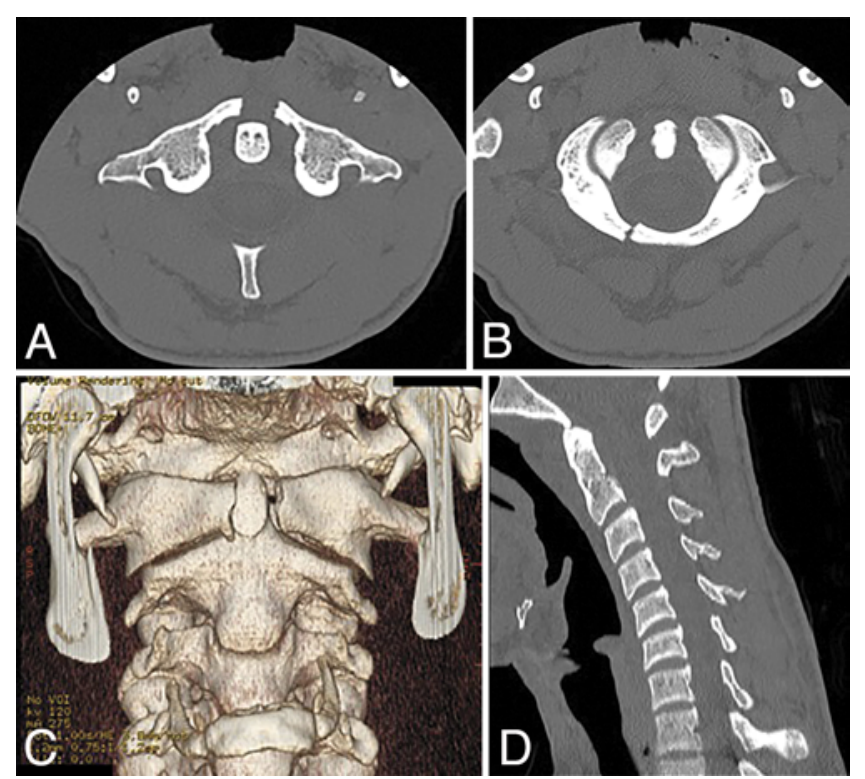

FIG. 1. A and B: Axial CT images demonstrating a widely displaced anterior arch fracture of $\mathrm{C}-1(\mathrm{~A})$ and a minimally displaced posterior arch fracture of $\mathrm{C}-1$ (B). C: Coronal 3D reconstruction of CT images demonstrating an anterior arch fracture of C-1. D: Sagittal CT image of the cervical spine, demonstrating characteristic loss of lordosis that is typical in football players with absence of the arch of C-1 due to a widely displaced anterior fracture. Figure is available in color online only.

reduction was desired, and thus a direct anterior approach was preferred. The loss of $40 \%-50 \%$ of lateral rotation that is the typical result of any $\mathrm{C} 1-2$ fusion technique was felt to be unacceptable to a defensive back in professional football. That position (more so than an offensive or defensive lineman, for example) requires wide surveys of the field from sideline to sideline and the need for full rotation to either side when turning to face an incoming pass or receiver. Thus, a motion-preserving technique like we describe would have a better chance of maintaining that necessary range of motion for his position. This third option was deemed the most likely to leave the patient with the stability and range of motion to potentially return to play.

\section{Operation}

The patient elected the third option. A single-stage transoral procedure was performed first. The patient was admitted preoperatively to the intensive care unit where Gardner Wells tongs were placed with an initial traction weight of $5 \mathrm{lbs}$, which was then increased to $10 \mathrm{lbs}$ in the hope that the fracture might close at least partially in preparation for ORIF. There was minimal change as determined by anteroposterior (AP) and lateral fluoroscopy. After 48 hours of traction, the patient was taken to the operating room. The procedure was performed under general anesthesia following an awake, fiber-optic intubation with a reinforced endotracheal tube. The patient was kept supine with his head resting on a foam doughnut. A Crowe-Davis oral retractor was placed. The uvula was resected, and 2 soft rubber catheters were passed through the nose and around the soft palate for retraction. A midline incision was made in the nasopharyngeal mucosa and 


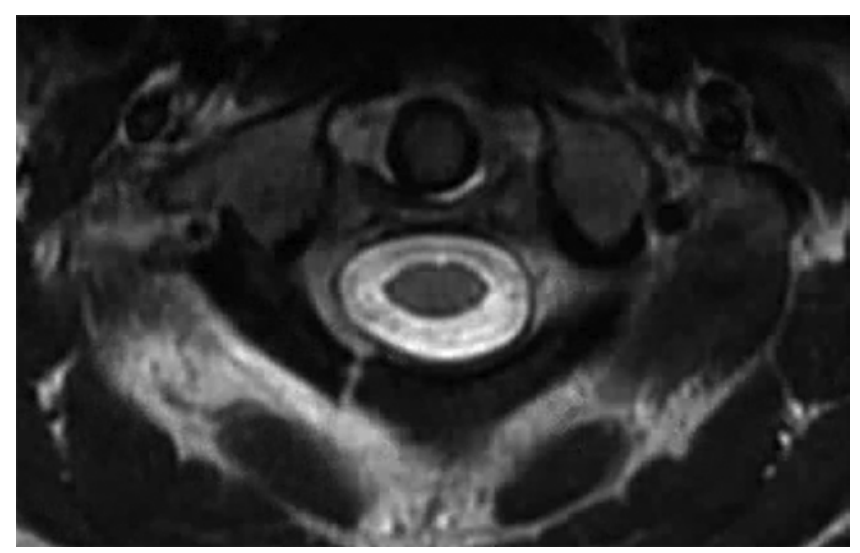

FIG. 2. Axial T2-weighted MR image demonstrating an intact transverse ligament.

extended down to the oropharynx. The superior pharyngeal constrictor muscle and paraspinous muscles were divided with monopolar cautery. With the widely displaced anterior arch fracture, a midline anterior tubercle of C-1 was not encountered; rather, the fractured ends of the arch were well off midline. A subperiosteal exposure of the entire arch out to the lateral masses bilaterally was required.

Two small divots were drilled into the anterior arch of $\mathrm{C}-1$ on both sides of the fracture using a $2-\mathrm{mm}$ bur. A long bone reduction ratcheting forceps engaged these divots and was used to successfully pull together the fractured ends of the anterior fracture. Unfortunately, the forceps location prevented applying a plate to the anterior arch for fixation. We therefore placed 2 small K-wires through an area of the C-1 arch that was not blocked by the reduction forceps. The K-wires were advanced into the dens of the odontoid. This maintained the reduction nicely and allowed for removal of the forceps and full access and visualization of the anterior arch of C-1. Using measurements derived from the preoperative CT scan, a customcontoured malleable reconstruction plate from the minifragment fracture set (6-hole plate) was affixed to the arch with the aid of 2.7-mm-diameter screws. Lateral fluoroscopy was used intermittently. Finally, 2 larger $3.5-\mathrm{mm}$ diameter, 20-mm-long screws were placed through the most lateral holes of the plate into the lateral masses of C-1. Anteroposterior (AP) images were difficult to obtain to guide the medial angulation; thus, this angulation was estimated from angles measured on the preoperative CT scan. Lateral fluoroscopy was helpful in trying to avoid violating the occiput-C1 joint. The final result was a nearly complete (less than a 1-mm gap visualized in one portion of the fracture site) reduction of the fracture.

The wound was irrigated with antibiotic solution, and powdered vancomycin was spread over the site. The muscle and mucosal layers were closed separately, and the site of the uvula resection in the soft palate was closed with Vicryl suture. The surgery was completed late in the afternoon, so the patient remained intubated until the next morning. He was extubated successfully and discharged from the hospital on postoperative Day 3 in a semirigid collar. Swallowing was normal in this patient within 3 weeks of recovery. Phonation was transiently weaker for

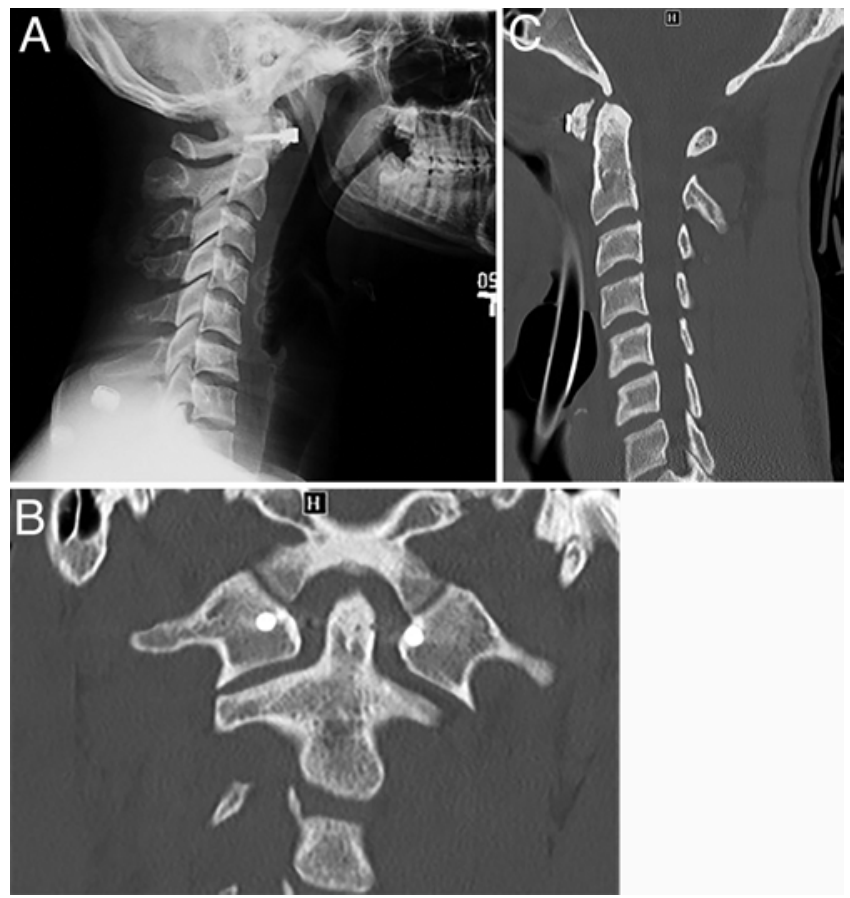

FIG. 3. Postoperative images. A: Lateral radiograph demonstrating successful transoral plating of the anterior C-1 arch fracture. B: Coronal CT image demonstrating successful instrumentation of the anterior $\mathrm{C}-1$ arch fracture with screws in the lateral masses of C-1. C: Sagittal CT image of the cervical spine, demonstrating successful reduction and instrumentation of the anterior $\mathrm{C}-1$ arch fracture.

a period of 2 weeks and then returned to normal. Postoperative plain radiographs and a CT scan confirmed successful reduction of the displaced anterior fracture (Fig. 3 ). The posterior arch fracture, however, was more widely displaced than preoperatively (Fig. 4). It was decided to allow for healing of both anterior and posterior fracture sites in a collar for 3-6 months. After 6 months, the patient had minimal neck pain and normal ranges of lateral rotation, flexion, and extension. CT images obtained at 6 months, however, showed a near-complete union of the anterior fixated fracture site but a nonunion of the displaced posterior arch fracture (Fig. 5).

A minimally invasive posterior ORIF of the displaced fracture site was recommended. Because of the chronic nature of the fracture, autologous cancellous iliac bone graft along with low-dose $(0.5 \mathrm{mg})$ bone morphogenetic protein (off-label use) was recommended. This procedure was performed in the prone position on chest rolls with the head held in the Mayfield head holder. A small incision was made over the posterior superior iliac spine. A 10 -mm opening was made in the superior cortex, and cancellous bone was harvested from the posterior iliac crest. For the approach to the posterior C-1 arch fracture, a 20-mm incision was made vertically in the midline, and a muscle-sparing, transmuscular approach to the arch was performed down to the fracture site. Great care was made not to interrupt tendinous insertion of the posterior musculature to the spinous process of C-2. A speculum retractor with a single-side blade (Microlumbar Discectomy Retractor) was used and provided excellent visualization. 


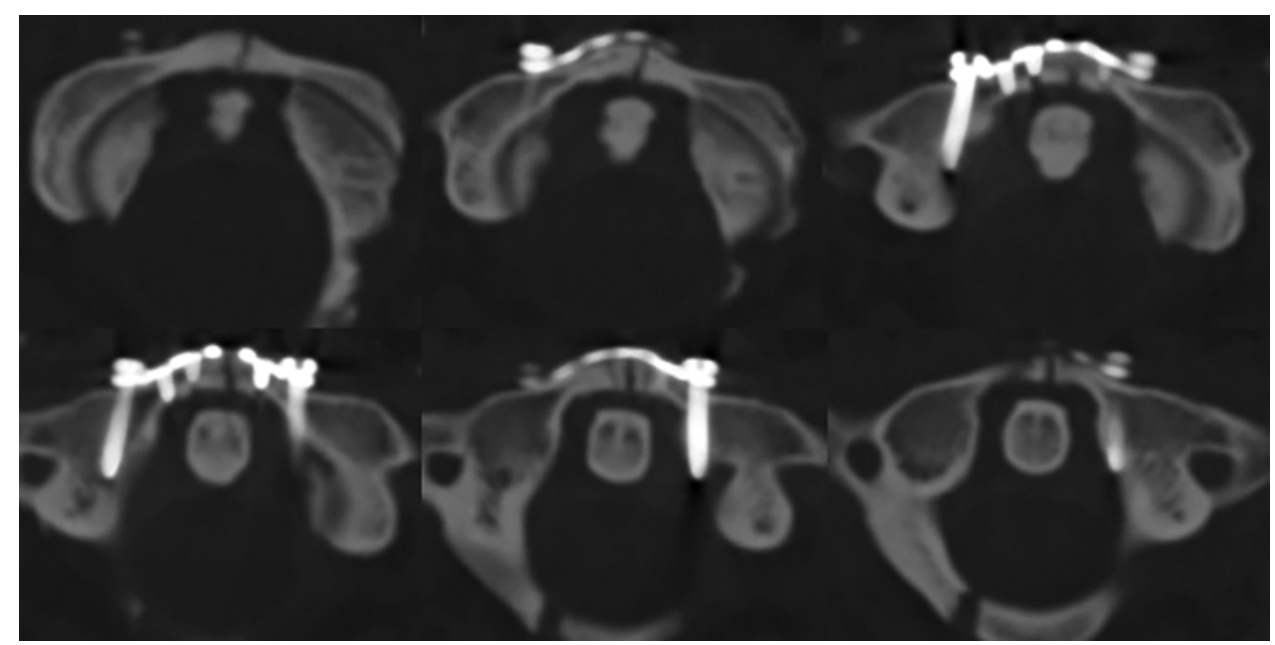

FIG. 4. Postoperative axial CT images demonstrating successful plating and reduction of the anterior C-1 arch fracture, with subsequent widening of the previously minimally displaced posterior C-1 arch fracture.

A subperiosteal exposure of the posterior arch and fracture was performed. The fibrous scar between the fracture edges was removed, and autologous iliac crest was used to fill the space along with $0.5 \mathrm{mg}$ of bone morphogenetic protein (half of an Extra-Extra-Small BMP-impregnated sponge: InFuse, Medtronic, Inc., off-label use). A smallfragment compression plate was then affixed to the posterior arch with screws. A standard 3-layer closure was then completed.

\section{Postoperative Course}

Within 5 months, a mature fusion across the posterior fracture site was confirmed on CT scanning. The majority of the anterior arch fracture healed, but not completely. AP, lateral, and flexion and extension cervical spine radiographs showed an atlanto-axial-dens interval (AADI) of $2-3 \mathrm{~mm}$ that increased to $4 \mathrm{~mm}$ with flexion (Fig. 6). A CT image of the cervical spine with rotational views was also obtained and demonstrated normal motion around the atlantoaxial complex. Furthermore, flexion and extension MR images without contrast were also obtained, which demonstrated a widely patent central spinal canal without spinal cord compression in all views. At the last follow-up ( $>1$ year since the posterior operation), the patient was doing well with minimal axial neck pain (1-year visual analog scale score for neck pain: 1-2/10 and no narcotic medications at 1 year) and full range of motion and no other signs or symptoms consistent with any postoperative complications.

\section{Discussion}

In addition to the advice provided by the treating surgeons, the player sought opinions from a wide variety of professional football league-affiliated orthopedic and neurosurgery team physicians as well as a non-National Football League (NFL)-affiliated neurosurgeon. Peerreviewed literature is lacking regarding return to play fol-

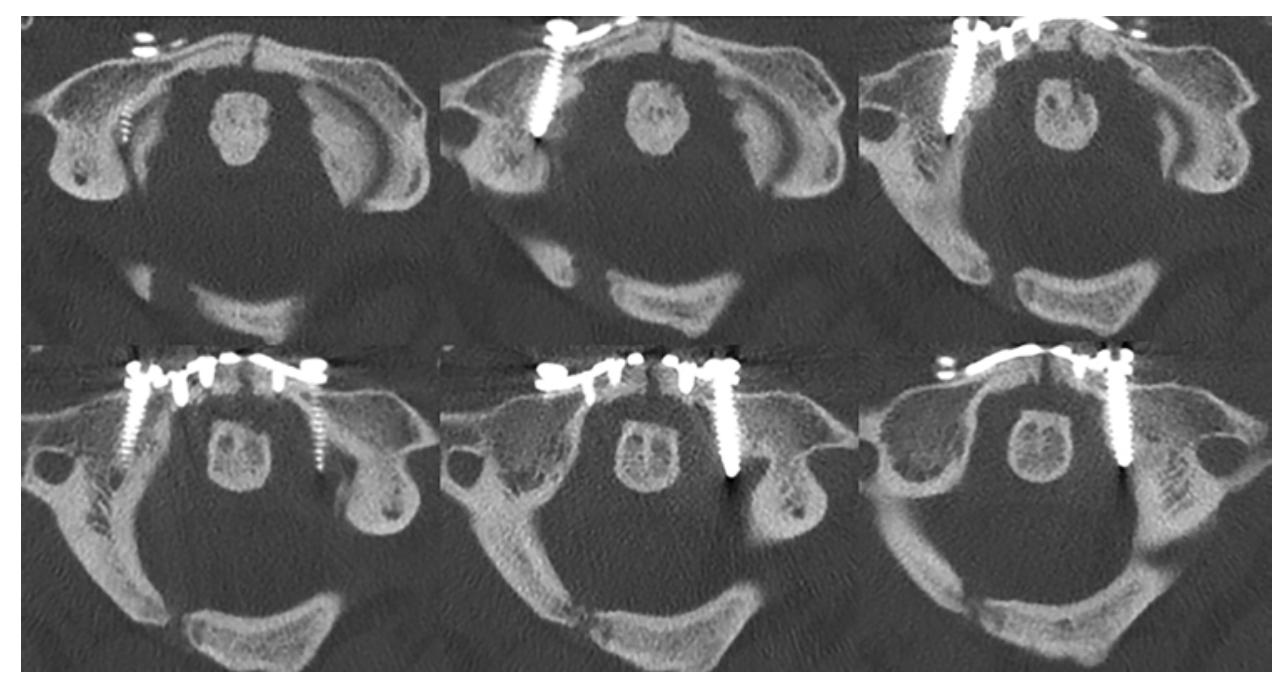

FIG. 5. Axial CT images obtained postoperatively, demonstrating a near-complete union of the anterior fixated fracture site but a nonunion of the displaced posterior arch fracture. 


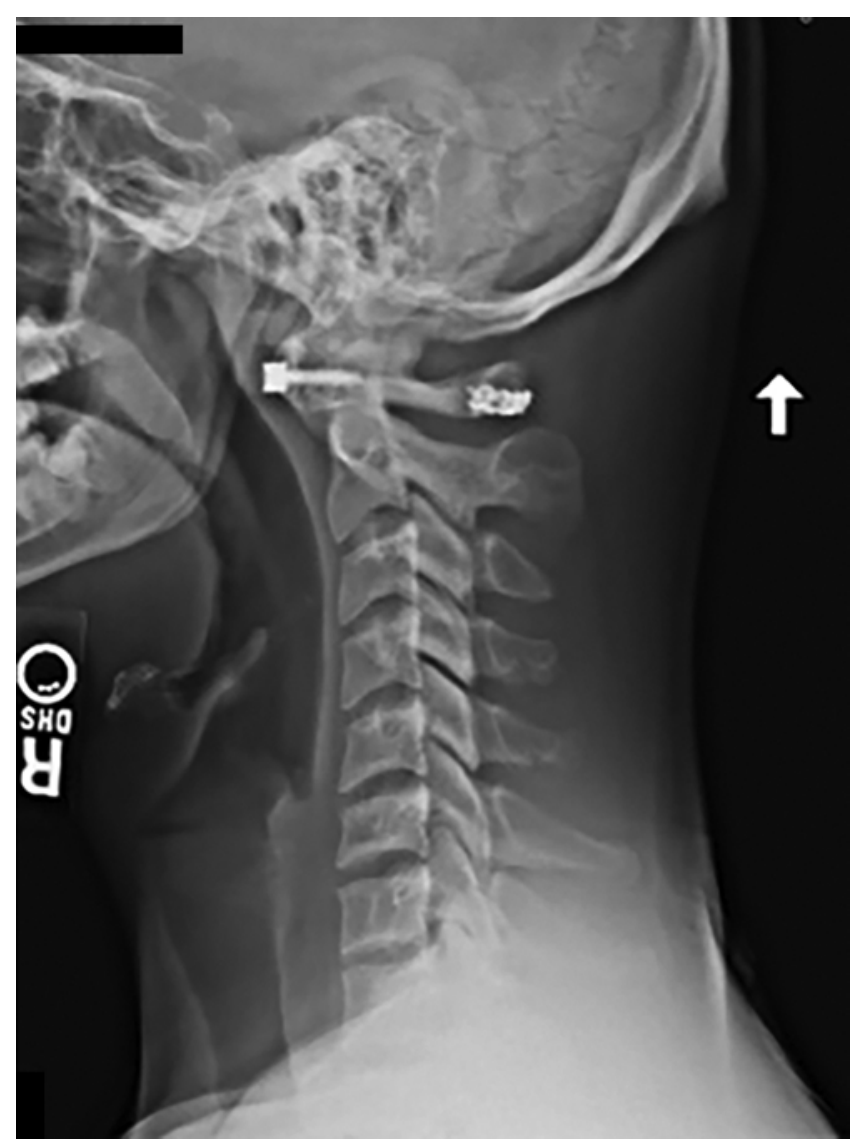

FIG. 6. Lateral radiograph obtained after the second operation, showing anterior and posterior plating and reduction of the C-1 arch fractures.

lowing any treatment of a Jefferson fracture in a professional football player or other type of athlete in a higherrisk contact sport. The general consensus was that the risks of returning to play could not be calculated. The patient clearly had developed some ligamentous laxity at C1-2. Although most people would be allowed to live an active life of recreation and athletics with an AADI that ranges between 2 and $4 \mathrm{~mm}$ between flexion and extension, the risks to a professional football player would very likely be higher. Furthermore, although the patient's posterior fracture healed $100 \%$ and the anterior fracture healed approximately $75 \%$, compared with the original defect, some question remained as to whether the status of his bone union alone increased the risk of failure and secondary injury to the C-1 fracture site in professional football. The player ultimately decided to not return to play as a professional football player.

Unstable, C-1 burst (Jefferson) fractures may be treated successfully in Halo or brace immobilization, or these fractures may be treated immediately with surgical stabilization. The standard treatment to date in patients with unhealed, unstable Jefferson fractures has been posterior C1-2 or occiput-C1-2 fusion. The consequences of these techniques include the obvious marked reduction in lateral rotation and, to a lesser extent, head tilt, flexion and extension, and lateral bending. Our experience with a professional football player in reducing the fracture initially via a transoral approach was challenging from a technical standpoint but did succeed ultimately in a satisfactory anterior reduction. The ORIF of the anterior displaced fracture, however, resulted in an increase in the displacement of the associated posterior arch fracture, ultimately resulting in the decision to perform a minimally invasive posterior arthrodesis in the hopes of returning the patient/ player to his profession. Although our patient did not return to play, he is now fully active with minimal neck discomfort and excellent range of motion. More experience is needed to evaluate whether this type of strategy could help not only professional or competitive amateur athletes, but also anyone who wishes to maintain maximal cervical range of motion and an active work, recreational, and athletic lifestyle.

\section{Acknowledgments}

We would like to thank the residents and fellows of the Emory University School of Medicine, Departments of Neurosurgery and Orthopaedic Surgery, for their excellent care of spine patients, such as the patient presented in this article.

\section{References}

1. Hu Y, Xu RM, Albert TJ, Vaccoro AR, Zhao HY, Ma WH, et al: Function-preserving reduction and fixation of unstable Jefferson fractures using a C1 posterior limited construct. J Spinal Disord Tech 27:E219-E225, 2014

2. Ruf M, Melcher R, Harms J: Transoral reduction and osteosynthesis $\mathrm{Cl}$ as a function-preserving option in the treatment of unstable Jefferson fractures. Spine (Phila Pa 1976) 29:823-827, 2004

3. Sun SH, Fang JL, Ma WH, Liu GY: [Transoral plate internal fixation for treatment of instability atlas fracture.] Zhongguo Gu Shang 26:81-84, 2013 (Chinese)

\section{Disclosures}

Dr. Rodts: royalty income from Medtronic, Inc., and Globus Medical, Inc. Dr. Heller: consultant for, royalties from, and stock share ownership in Medtronic. These activities are not related to the subject of this manuscript.

\section{Author Contributions}

Conception and design: Rodts, Heller. Acquisition of data: all authors. Analysis and interpretation of data: Baum, Heller. Drafting the article: Rodts. Critically revising the article: Baum, Heller. Reviewed submitted version of manuscript: Baum. Administrative/technical/material support: Baum. Study supervision: Heller.

\section{Correspondence}

Gerald E. Rodts Jr., The Emory Orthopaedics \& Spine Center, Emory University School of Medicine, 59 Executive Park South, Ste.3000, Atlanta, GA 30329. email: grodts@emory.edu. 